\title{
Partial phenotype conversion and differential trait response to conditions of husbandry in mice
}

\author{
Julia Brenmoehl ${ }^{1} \cdot$ Christina Walz $^{1} \cdot$ Marion Spitschak ${ }^{1} \cdot$ Elisa Wirthgen $^{1} \cdot$ Michael Walz $^{1} \cdot$ Martina Langhammer $^{2}$. \\ Armin Tuchscherer ${ }^{2} \cdot$ Ronald Naumann ${ }^{3} \cdot$ Andreas Hoeflich $^{1}$
}

Received: 24 May 2017 / Revised: 22 November 2017 / Accepted: 27 November 2017 / Published online: 6 December 2017

(c) The Author(s) 2017. This article is an open access publication

\begin{abstract}
Functional genome analysis usually is performed on the level of genotype-phenotype interaction. However, phenotypes also depend on the relations between genomes and environment. In our experimental system, we observed differential response to environmental factors defined by different conditions of husbandry in a semi-barrier unit or in a SPF (specific pathogen free) barrier unit, which resulted in partial reversal of phenotypes previously observed under semi-barrier conditions. To provide an update of basic phenotypes in unselected and randomly mated controls (DUC) and long-term selected DUhTP (Dummerstorf high treadmill performance) mice in the SPF facility, we compared growth parameters, reproductive performance, the accretion of muscle and fat mass, physical activity, and running performance as well as food intake in all experimental groups. For selected parameters, the comparative analysis spans more than 30 generations. In DUC mice, under SPF conditions a more than threefold $(P<0.0001)$ higher subcutaneous fat mass, higher muscle mass by about $25 \%$ $(P<0.0001)$, but lower epididymal fat mass in DUhTP mice by about $20 \%(P<0.0001)$ were observed. In SPF husbandry, body weight increased to a stronger extent in adult DUC mice $(\approx 20 \% ; P<0.0001)$ than in DUhTP mice $(\approx 8 \% ; P=0.001)$. The concentrations of IGF-1 and IGFBPs in the serum as well as the liver weights were similar in all experimental groups, indicating growth effects independent of the somatotropic axis. Under SPF conditions the litter size at birth increased in DUC mice $(P<0.001)$ but not in DUhTP mice. The differential effect of husbandry on body weights at day 21 and concentrations of triglycerides in the serum of our model were due to the different diets used in the semi-barrier and in the SPF facility. Our results demonstrate differential trait response to environmental factors resulting in partial phenotype conversion in our experimental system. The existence of conditional phenotypes as a result of genotype-environment interactions points to the importance of environmental factors in functional genome analysis.
\end{abstract}

Keywords Semi-barrier husbandry $\cdot$ SPF husbandry $\cdot$ Body weight $\cdot$ Non-inbred mouse line $\cdot$ Standard chow $\cdot$ Phenotype conversion

\section{Introduction}

Communicated by G. Heldmaier.

Andreas Hoeflich

hoeflich@fbn-dummerstorf.de

1 Institute for Genome Biology, Leibniz-Institute for Farm Animal Biology (FBN), Wilhelm-Stahl-Allee 2, 18196 Dummerstorf, Germany

2 Institute Biometry and Genetics, Leibniz-Institute for Farm Animal Biology (FBN), Wilhelm-Stahl-Allee 2, 18196 Dummerstorf, Germany

3 Max Planck Institute of Molecular Cell Biology and Genetics, Pfotenhauerstraße 108, 01307 Dresden, Germany
Since 1983 in Dummerstorf a high treadmill performance mouse model (DUhTP) had been established for the analysis of energy metabolism applying long term phenotype selection for high treadmill performance (Dietl et al. 2004). Selection was performed in the absence of running wheels within the home cage to exclude self-training effects within the selection response. The dimension of the selection experiment is remarkable, because selection period took more than 30 years and included up to 100 families per generation. The initial population of the Dummerstorf outbred mouse lines was a systematic crossbred of four inbred and four outbred founder mouse strains starting in the 1970s (Falkenberg 
et al. 2000). Both the unselected and randomly mated control line (DUC) and the DUhTP originate from this genetic founder pool. Trait selection in DUhTP was based on a singular submaximal endurance exercise test on a treadmill at day 70 of life (Falkenberg et al. 2000). Without previous training, DUhTP mice cover fourfold increased distances if compared to DUC mice (Brenmoehl et al. 2013). Interestingly, subcutaneous adipose tissue of DUhTP mice is characterized by elevated concentrations of metabolic enzymes for lipid synthesis (fatty acid synthase, acetyl-CoA carboxylase) but also for lipid hydrolysis (hormone-sensitive lipase) or oxidation (long-chain acyl dehydrogenase), respectively, as well as elevated levels of mitochondrial proteins arguing for high mitochondrial biogenesis (Brenmoehl et al. 2015). In response to moderate voluntary physical activity over a period of 3 weeks in running wheels, concentrations of inducer of mitochondrial biogenesis are further increased resulting in efficient fat mobilization in DUhTP mice (Brenmoehl et al. 2015). Recently, we identified genetic linkage of fat cell browning and metabolic health, because young male DUhTP mice are characterized by increased tolerance against oral glucose (Brenmoehl et al. 2016). Under semibarrier conditions, sedentary DUhTP mice are characterized by increased accumulation of body fat compared to DUC mice (Brenmoehl et al. 2015). After hygienic reorganization of both lines from the semi-barrier to a new SPF barrier unit, we observed differential responses for the new environment resulting in the partial reversal of phenotype, which prompted us to compare isolated phenotypes observed in both mouse facilities. Because we used different diets in the semi-barrier and in the SPF facility, we also assessed the effects of both commercial diets on body weight under identical husbandry conditions.

\section{Materials and methods}

\section{Animals and husbandry}

All in vivo experiments were performed in accordance with national and international animal protection guidelines [German Animal Welfare Act (TierSchG)] and were approved by our internal institutional review board. The study was conducted at the mouse animal facility of the Leibniz Institute for Farm Animal Biology in Dummerstorf, Germany. We used a non-inbred mouse line that has been generated by selection over 130 generations for high treadmill performance (DUhTP) (Falkenberg et al. 2000) and the control mice (DUC) that had been generated from the identical base population without phenotype selection (Dietl et al. 2004). Body weights were simultaneously considered from animals between generations 103 and 137 (DUhTP; each $n>23$ ) and between 147 and 182 (DUC; each $n>87$ ). For both lines, littermates were registered for every generation and standardized to a number of eight pups per female in the semi barrier unit and to ten pups per mother immediately after birth in the SPF unit. Animals were housed in Makrolon-cages Type II (EBECO, Castrop-Rauxel, Germany) in a semi-barrier system like described recently (Brenmoehl et al. 2013). In the SPF barrier unit, the mice were kept in polysulfone cages of $267 \times 207 \times 140 \mathrm{~mm}$ (H-Temp PSU, Type II, Eurostandard Tecniplast, Germany). Hygiene management and health monitoring in SPF husbandry were performed according to the recommendations of the FELASA (Mahler Convenor et al. 2014). In both conditions of mouse husbandry, environmental conditions were defined by a 12-h light-dark cycle (room temperature $22.5 \pm 0.2{ }^{\circ} \mathrm{C}$, humidity $40-60 \%$ ) and the animals had free access to pellet concentration and water. Semi-barrier animals received food \#1314 from Altromin (Lage, Germany). Mice in SPF conditions were fed with autoclaved Ssniff ${ }^{\circledR}$ M-Z food (Ssniff-Spezialdiäten $\mathrm{GmbH}$, Soest, Germany; Table 1). For the resettlement of the new SPF mouse facility in Dummerstorf, by vitrification cryopreserved embryos from individual DUC mice were transferred into pseudopregnant $\mathrm{Crl}$ :CD1(ICR) fosters in an existing SPF facility (Max Planck Institute of Molecular Cell Biology and Genetics, Dresden, Germany). New generated $\mathrm{DUC}_{\mathrm{SPF}}$ mice of generation 150 were then imported to the empty SPF facility in Dummerstorf and expanded as the initial base population. After verification of the SPF status in the new barrier unit (Gesellschaft für innovative Mikroökologie mbH, Michendorf, Germany), fresh recovered embryos from DUhTP $_{\mathrm{SB}}$ and $\mathrm{DUC}_{\mathrm{SB}}$ mice were fresh-transferred to pseudopregnant $\mathrm{CD} 1$ foster females and $\mathrm{DUC}_{\mathrm{SPF}}$ mice.

Voluntary physical activity and food consumption in both mouse lines were recorded at an age of 49-70 days using running wheels $(d=33.4 \mathrm{~cm}$; Tecniplast, Hohenpeißenberg, Germany). In the same period, food consumption was monitored in animals of DUhTP (semi-barrier: generation 105; SPF: generation 128 and 135) and DUC mice (semibarrier: generation 149; SPF: generation 173 and 180). For

Table 1 Composition of used food

\begin{tabular}{lll}
\hline & Altromin \#1314 & Ssniff M-Z \\
\hline Dry matter & $89.0 \%$ & $88.0 \%$ \\
Crude protein & $22.5 \%$ & $22.0 \%$ \\
Crude fat & $5.0 \%$ & $4.5 \%$ \\
Crude fiber & $4.5 \%$ & $3.9 \%$ \\
Crude ash & $6.5 \%$ & $6.7 \%$ \\
Nitrogen-free extracts & $50.5 \%$ & $50 \%$ \\
Metabolized energy & $2988 \mathrm{kcal} / \mathrm{kg}$ & $3272 \mathrm{kcal} / \mathrm{kg}$ \\
Calories from protein & $27 \%$ & $36 \%$ \\
Calories from fat & $13 \%$ & $11 \%$ \\
Calories from carbohydrates & $60 \%$ & $53 \%$ \\
\hline
\end{tabular}


the examination of body composition of DUhTP and DUC mice, data from generation 93, 95, 105, $118\left(\mathrm{DUhTP}_{\mathrm{SB}}\right)$,

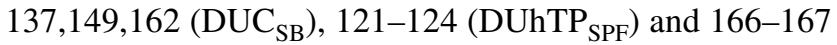
$\left(\mathrm{DUC}_{\mathrm{SPF}}\right)$ were used. Body length determination was performed at day 42 with mice of generation $100\left(\mathrm{DUhTP}_{\mathrm{SB}}\right)$, $144\left(\mathrm{DUC}_{\mathrm{SB}}\right), 137$ (DUhTP $\left.\mathrm{SPF}\right)$ and $182\left(\mathrm{DUC}_{\mathrm{SPF}}\right)$. All mice were killed at day 70 of age via decapitation for blood sampling and tissues (liver, Musculus rectus femoris, epididymal fat, and isolated subcutaneous fat (generation 105, 121-124, $149,167)$ ) were weighted, snap-frozen in liquid nitrogen, and stored at $-70{ }^{\circ} \mathrm{C}$ for subsequent analysis. Serum was stored at $-20^{\circ} \mathrm{C}$. For the longitudinal analysis of body mass after weaning, we analyzed animals of generation 134 $\left(\right.$ DUhTP $\left._{\mathrm{SPF}}\right)$ and $179\left(\mathrm{DUC}_{\mathrm{SPF}}\right)$. We included $30\left(\mathrm{DUC}_{\mathrm{SPF}}\right)$ and $15\left(\right.$ DUhTP $\left._{\mathrm{SPF}}\right)$ standardized litters $(n=10)$, respectively. Furthermore, 30 males and 16 females of DUhTP ${ }_{\mathrm{SPF}}$ in generation 135 and $\mathrm{DUC}_{\mathrm{SPF}}$ mice in generation 180 were divided and afterwards kept for two generations as an own subpopulation without selection procedure (marked as generation $135^{*}-137^{*}$ and $180^{*}-182^{*}$, respectively). Mice in the parallel husbandry were kept in individually ventilated cages (GM500 Mouse IVC Green Line, Tecniplast, Germany) and received either "semi-barrier food" from Altromin or "autoclaved SPF-food" from Ssniff and were analyzed for body masses and food consumption.

\section{Analysis of the IGF system}

Serum concentrations of IGF-1 were analyzed using the specific ELISA for mouse and rat IGF-1 (E25, Mediagnost, Reutlingen, Germany) according to the manufacturer's instruction. The sensitivity of the assay system was $<0.029 \mathrm{ng} / \mathrm{ml}$ and the analytical range for recombinant IGF-1 was $0.5-18 \mathrm{ng} / \mathrm{ml}$. The intra- and inter-assay coefficients of variation (CV\%) were 4.2-5.9 and 5.3-6.7\%, respectively.

Serum IGFBP-2, -3 , and -4 were analyzed by quantitative Western ligand blot analysis as described previously (Wirthgen et al. 2016). Briefly, serum samples were diluted 1:20, boiled in sample buffer $(312.5 \mathrm{mM}$ Tris at $\mathrm{pH} 6.8,50 \%(\mathrm{w} / \mathrm{v})$ glycerol, $5 \mathrm{mM}$ EDTA, $1 \%(\mathrm{w} / \mathrm{v})$ SDS, and $0.02 \%$ bromophenol blue) for $5 \mathrm{~min}$. Proteins were separated by $12 \%$ SDS-PAGE followed by the transfer onto a polyvinylidene fluoride membrane (Millipore, Bedford, USA). The blots were blocked and then incubated with biotin-labeled human IGF-2 (1:500; BioIGF2-10; ibt-systems, Binzwangen, Germany). The IGF-binding proteins were detected by enhanced chemiluminescence using LuminataTM Forte (Millipore, Bedford, USA). Bands were visualized on KODAK Image Station 4000MM (Molecular Imaging Systems, Carestream Health, Inc., New Haven, USA) and quantified using Gelanalyzer2010a software. Signal intensities were corrected for background and quantified using human recombinant standards (IGFBP-2 to -4 , R\&D Systems, WiesbadenNordenstadt, Germany) diluted in artificial serum matrix (Biopanda, County Down, United Kingdom) as calibrators on each blot. The calculation of the IGFBP concentrations in plasma was performed using GraphPad Prism6 software and corrected for dilution and volume/lane of each sample. The analytical range for each IGFBP was $150-15000 \mathrm{ng} / \mathrm{ml}$.

\section{Analysis of serum triglycerides}

Triglycerides (TG) were assayed in serum samples using a commercial kit (No. LT-TR 0015; Labor \& Technik Eberhard Lehmann, Berlin, Germany).

\section{Statistical analysis}

Data analysis was performed using SAS software (Version 9.4 for Windows, SAS Institute Inc., Cary, NC, USA). Descriptive statistics and tests for normality were calculated with the UNIVARIATE procedure of Base SAS software (SAS Institute Inc. 2013. Base SAS ${ }^{\circledR} 9.4$ Procedures Guide, Second Edition. Cary,NC: SAS Institute Inc.). Data considered as approximately normal were analyzed by ANOVA using the MIXED procedure of SAS/STAT software (SAS Institute Inc. 2013. SAS/STAT ${ }^{\circledR} 13.1$ User's Guide. Cary, NC: SAS Institute Inc.). The ANOVA model for the 70d data contained the fixed factors line (levels: DUC, DUhTP), group (levels SB, SPF) and the interaction line $\times$ group. Homogeneity of covariance parameters across groups was tested for all ANOVA models. In addition, least-squares means (LSM) and their standard errors (SE) were computed for each fixed effect in the models, and all pairwise differences of LS-means were tested by the Tukey-Kramer procedure. Effects and differences were considered significant if $P<0.05$.

\section{Results}

\section{Body mass as a function of animal husbandry}

The mouse models DUC and DUhTP were established under semi-barrier conditions starting in the early 1970s and 1980s, respectively. In 2012, all mouse lines were transferred from the semi-barrier unit to the newly built mouse facility with standardized environmental and working procedures defined by the SPF status. Longitudinal analysis of body mass in DUC and DUhTP mice revealed positive effects of SPF husbandry only in DUC but not in DUhTP mice (Fig. 1). At an age of 21 days, $\mathrm{DUC}_{\mathrm{SPF}}$ mice were heavier by $36.4 \%(P<0.0001)$ if compared to DUC mice in the semibarrier (Fig. 1a). Also, at an age of 42 days, $D_{\mathrm{SPF}}$ mice were characterized by elevated body mass if compared to 
Fig. 1 Body mass and body length of male DUhTP (grey data points) and DUC mice (black data points) kept under semi-barrier or SPF conditions. Body mass is presented for an age of 21 days (a) and 42 days after birth (b). Body lengths were recorded at day 42 in both mouse lines (c). Animals were kept in semi-barrier (dots) and SPF husbandry (triangles), respectively. Data are presented as means $\pm \mathrm{SE}$ $(* P<0.05, \S P<0.0001)$

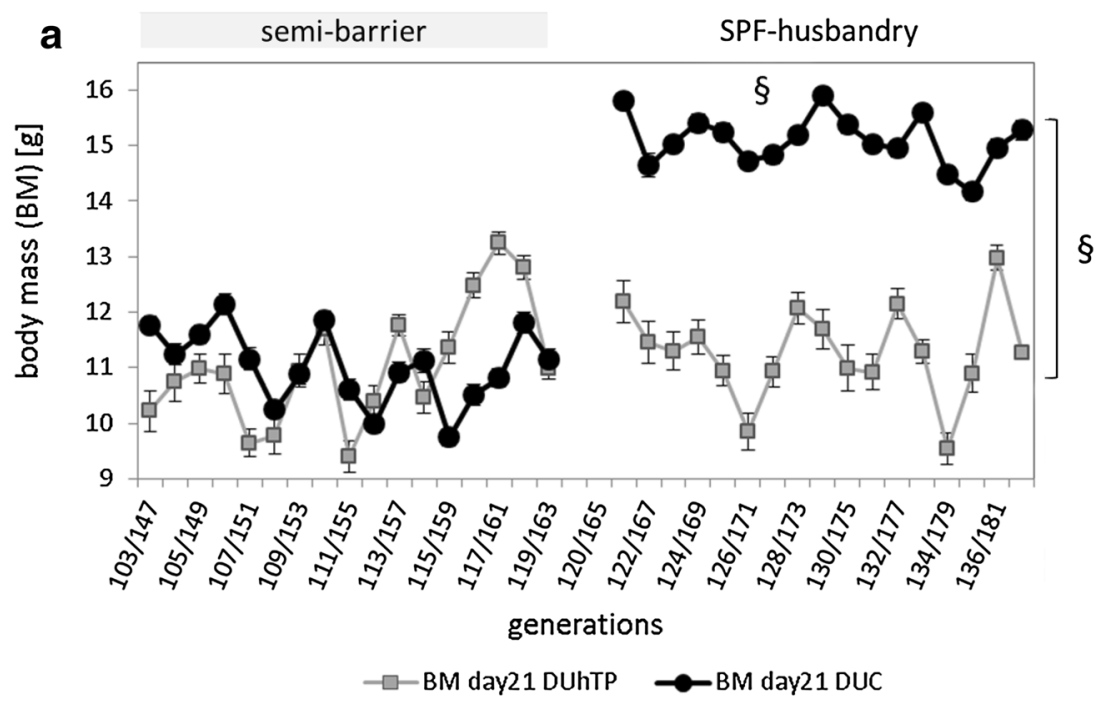

b
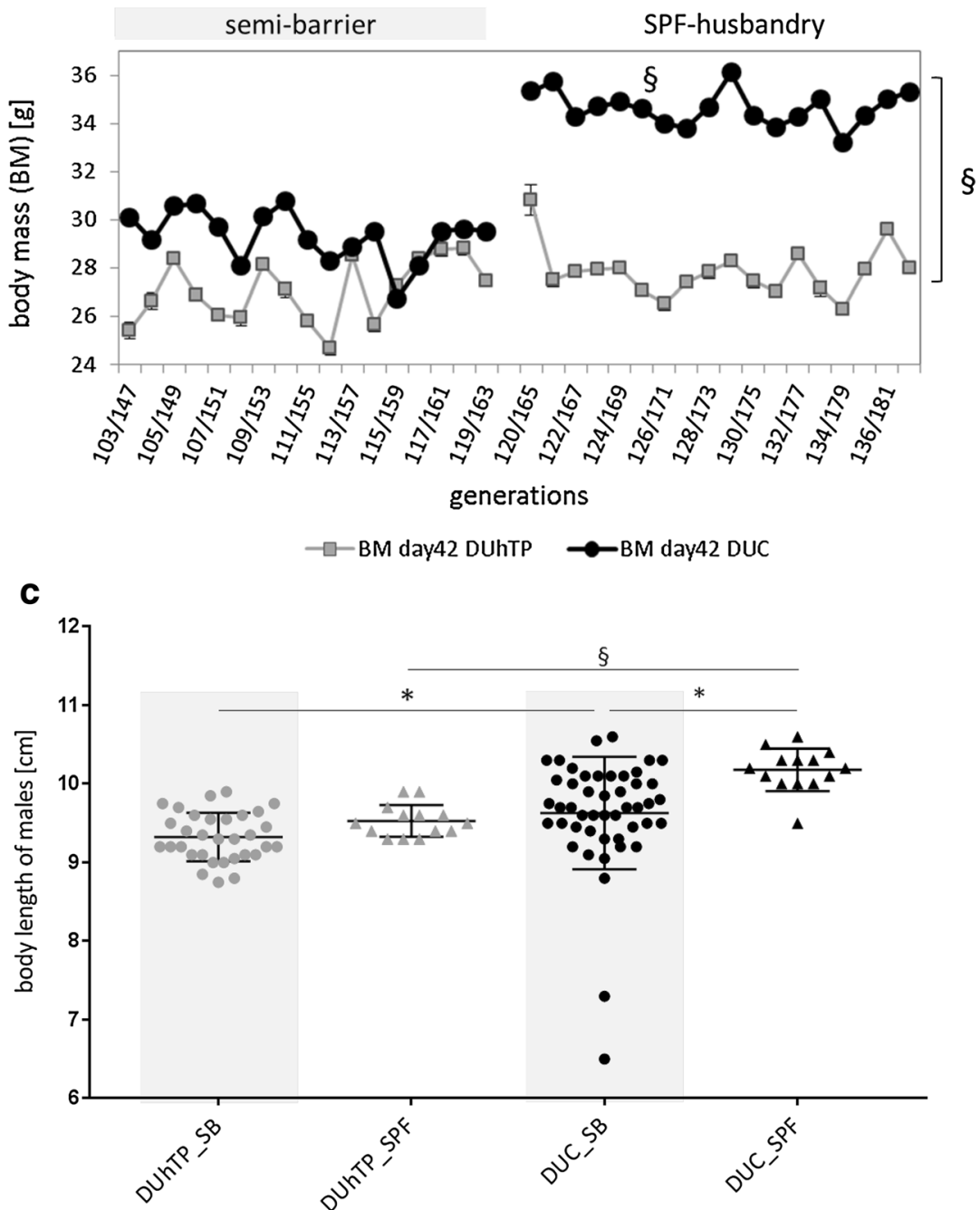

DUC mice kept in the semi-barrier $(+18 \% ; P<0.0001$; Fig. 1b). By contrast, both conditions of husbandry did not impact on body mass of DUhTP mice at an age of 21 or
42 days. Under SPF conditions, DUC mice also were significantly taller than mice of the same line in SB husbandry $(10.18 \pm 0.072$ versus $9.63 \pm 0.104 \mathrm{~cm}, P<0.01)$ while the 
body lengths of DUhTP mice were similar in both facilities (Fig. 1c). Consequently, body lengths differed between both mouse lines in SPF husbandry by more almost $7 \%$ $(P<0.0001)$. Under semi-barrier conditions, DUhTP mice were smaller by only $3 \%(P<0.05)$.

At birth, SPF conditions increased body weights in both mouse lines (DUC $+3.3 \%$; DUhTP $+6.9 \%$; both $P<0.0001$; Fig. 2a). The body weights of DUhTP mice were higher at birth if compared to DUC mice (BM0 in DUhTP $1.95 \mathrm{~g} \pm 0.03$; BM0 in DUC $1.81 \mathrm{~g} \pm 0.02 ; P<0.0001$; Fig. 2a). Interestingly, litter size was significantly increased by SPF husbandry exclusively in DUC mice (DUC +1.07 littermate, $P<0.0001$; Fig. 2b), which further increased the difference of litter size between $\mathrm{DUC}_{\mathrm{SPF}}$ and DUhTP $\mathrm{SPF}$ mice $(+13.7 \%, P<0.0001)$.

\section{Postnatal weight gain}

Longitudinal assessment of body mass in SPF husbandry revealed higher body weights in $\mathrm{DUC}_{\mathrm{SPF}}$ versus $\mathrm{DUhTP}_{\mathrm{SPF}}$ mice shortly between days 6 and 21 after birth (Fig. 3a). DUC $_{\mathrm{SPF}}$ offspring has significantly increased level of postnatal weight gain immediately after birth prolonging the whole suckling period. In fact, postnatal weight gain from birth to day 21 identified significantly higher mass accretion in DUC $_{\mathrm{SPF}}$ offspring if compared to DUhTP ${ }_{\mathrm{SPF}}$ mice (Fig. 3b).

\section{Differential effects of animal husbandry on organ weights}

At an age of 70 days under semi-barrier conditions, body and liver weights were higher in $\mathrm{DUC}_{\mathrm{SB}}$ mice $(P<0.01)$, whereas epididymal and subcutaneous fat depots revealed decrease weights $(P<0.001)$ if compared to DUhTP ${ }_{\mathrm{SB}}$ mice (Table 2). Under SPF conditions, higher body and liver weights also were observed in $\mathrm{DUC}_{\mathrm{SPF}}$ mice $(P<0.001)$. However, the increases of body weights in $\mathrm{DUC}_{\mathrm{SPF}}$ mice were much higher if compared to semi-barrier husbandry $(P<0.0001)$. In DUC mice, maintained under SPF conditions a more than threefold increase of subcutaneous fat
Fig. 2 Birth weight (a) and litter size (b) in DUhTP (grey data points) and DUC mice (black data points) kept under semibarrier or SPF conditions. Data are presented as means $\pm \mathrm{SE}(\S$ $P<0.0001)$
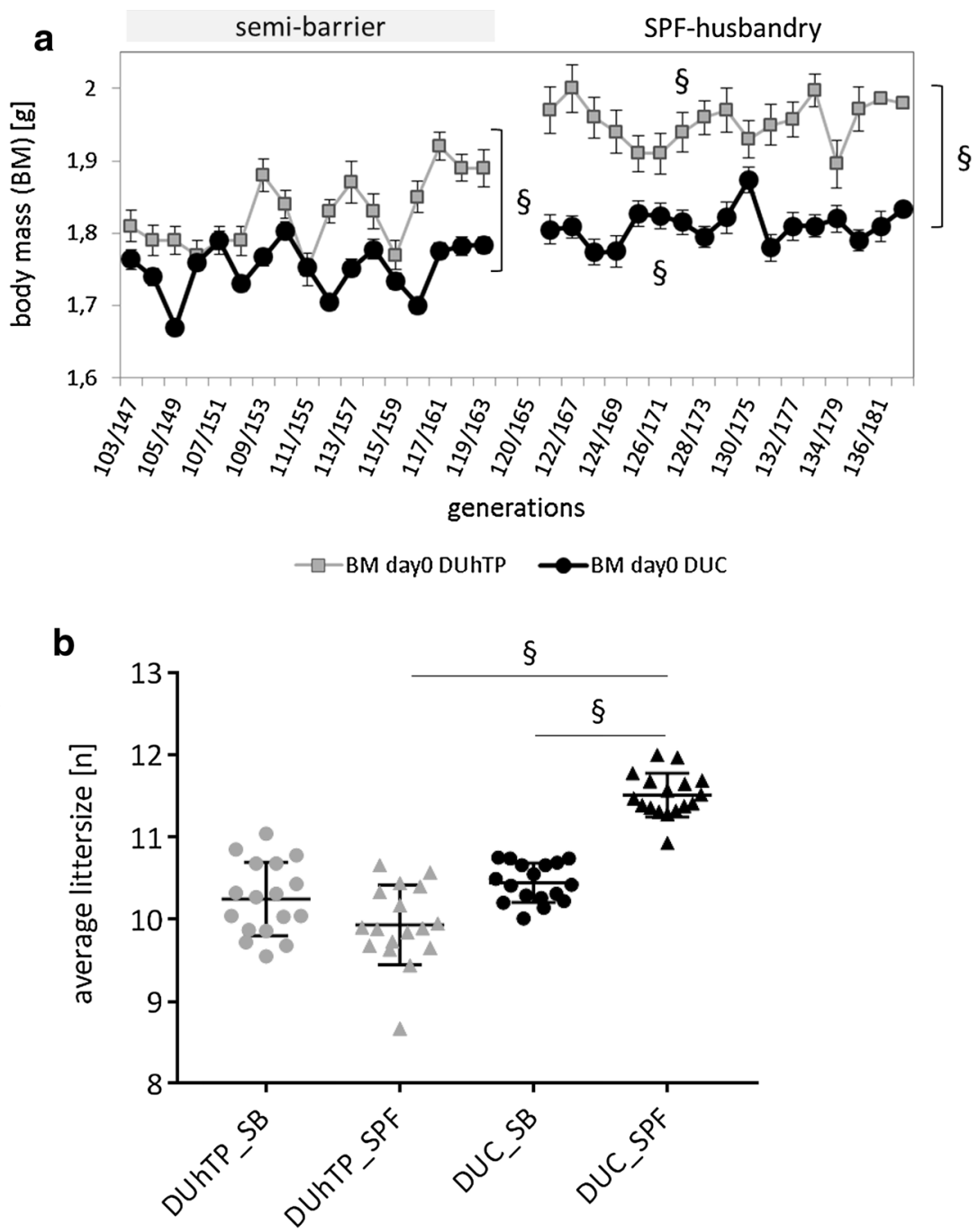
Fig. 3 Postnatal weight gain in DUhTP (grey data points) and DUC mice (black data points) kept under SPF conditions (a) and effect of animal husbandry on postnatal weight gain (b) in both mouse lines. For the comparison of weight gain, animals of individual generations were kept in semi-barrier (dots) and SPF husbandry (triangle), respectively. One data point represents one generation. Body mass of litter size-standardized pups $(n=10)$ between day 0 and 21 after birth was determined in DUC ( $n=30$, generation 134) and DUhTP $(n=15$, generation 179) littermates. Data are presented as means \pm SE $(*$ $P<0.05, \S P<0.0001)$ a

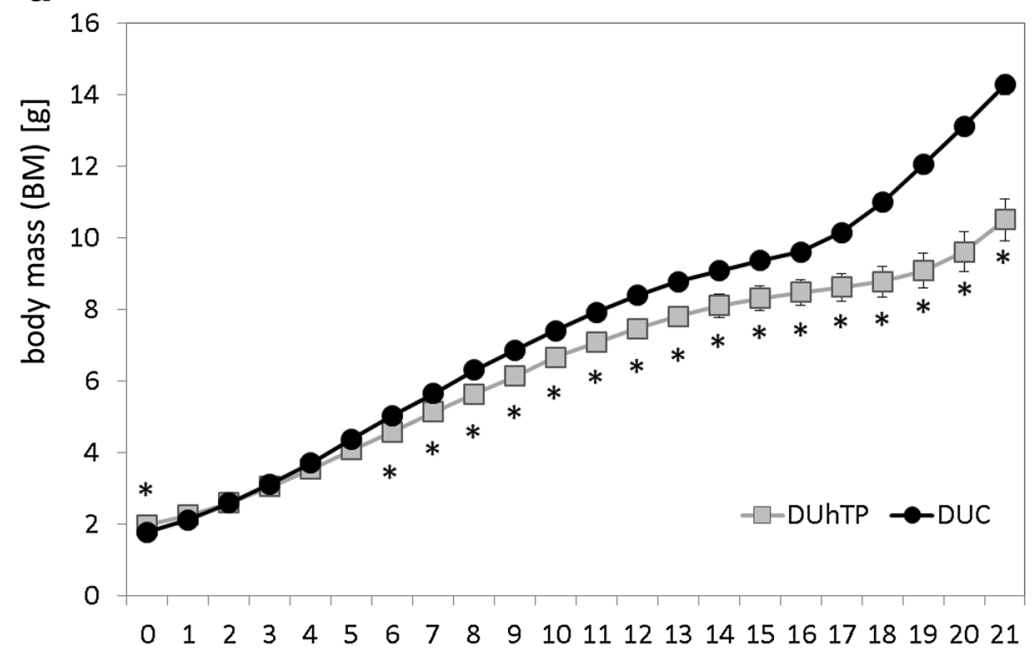

LT

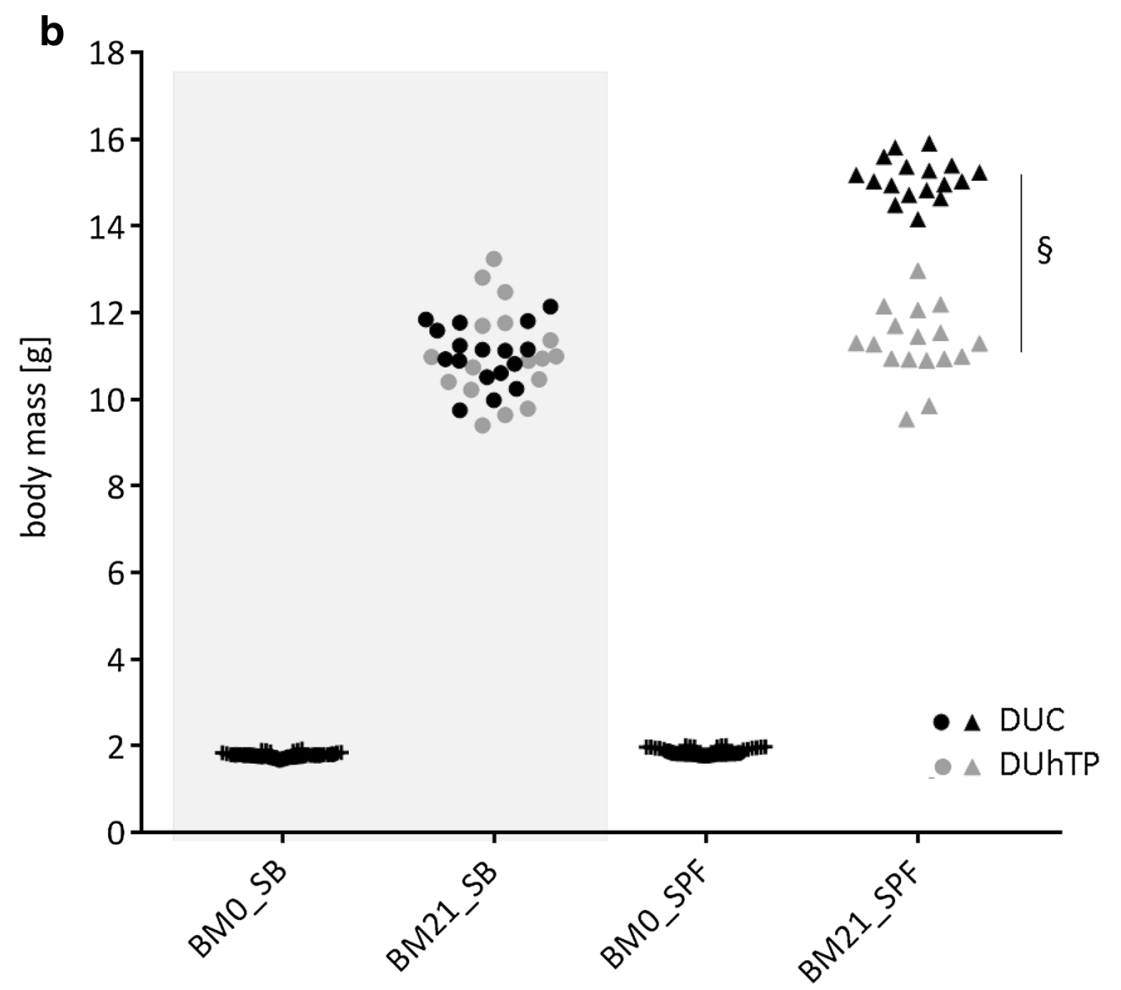

mass was observed if compared to semi-barrier husbandry

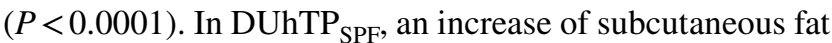
by only $25 \%$ was found if kept under SPF conditions (not significant). The increase of epididymal fat by about $30 \%$ only has borderline significance $(P=0.067)$. By contrast, in DUhTP $_{\text {SPF }}$ mice epididymal fat mass was decreased if kept under SPF conditions $(-30 \% ; P<0.0001)$. Thus, DUhTP mice, characterized by higher fat mass under semi-barrier conditions $(P<0.001)$, have lower fat mass under SPF conditions $(P<0.001)$ if compared to $\mathrm{DUC}_{\mathrm{SPF}}$ mice.
Generally, while control mice $\mathrm{DUC}_{\mathrm{SB}}$ in semi-barrier husbandry were characterized by decreased epididymal and subcutaneous fat in contrast to $\mathrm{DUhTP}_{\mathrm{SB}}$ mice, they show now higher fat masses than DUhTP $_{\mathrm{SPF}}$ mice.

In response to SPF husbandry, concentrations of serum TG were decreased in DUhTP $(P<0.0005$; Fig. 4$)$ and increased in DUC mice $(P<0.05)$. In $\mathrm{SB}$ conditions, DUhTP mice were characterized by higher TG level when compared to controls $(P<0.05)$ while under SPF conditions DUC mice had higher TG concentrations than DUhTP mice $(P<0.0001)$. 
Table 2 Overview of body masses and tissue weights of 70 day old male DUhTP and DUC mice from individual generations

\begin{tabular}{|c|c|c|c|c|c|c|c|c|c|}
\hline & $n$ & DUhTP_SB & DUhTP_SPF & $P$ & DUC_SB & DUC_SPF & $P$ & $\begin{array}{l}P \text { (DUhTP_SB } \\
\text { versus DUC_ } \\
\text { SB) }\end{array}$ & $\begin{array}{l}P(\text { DUhTP_SPF } \\
\text { versus DUC_SPF })\end{array}$ \\
\hline Body mass (g) & $>24$ & $30.14 \pm 0.60$ & $32.61 \pm 0.30$ & 0.00111 & $33.93 \pm 0.54$ & $40.52 \pm 0.63$ & $<0.00001$ & 0.00002 & $<0.00001$ \\
\hline $\begin{array}{l}\text { M. rectus femoris } \\
(\mathrm{g})\end{array}$ & $>24$ & $0.41 \pm 0.01$ & $0.38 \pm 0.01$ & 0.10401 & $0.39 \pm 0.01$ & $0.49 \pm 0.01$ & $<0.00001$ & 0.69878 & $<0.00001$ \\
\hline Liver mass (g) & $>24$ & $1.73 \pm 0.05$ & $1.74 \pm 0.02$ & 0.99655 & $1.96 \pm 0.05$ & $1.97 \pm 0.04$ & 0.99983 & 0.00143 & 0.00084 \\
\hline $\begin{array}{l}\text { Subcutaneous } \\
\text { fat }(\mathrm{g})\end{array}$ & $>10$ & $0.36 \pm 0.03$ & $0.45 \pm 0.02$ & 0.44078 & $0.20 \pm 0.01$ & $0.68 \pm 0.06$ & $<0.00001$ & 0.00003 & 0.00006 \\
\hline Epidymal fat (g) & $>21$ & $0.43 \pm 0.02$ & $0.24 \pm 0.01$ & $<0.00001$ & $0.29 \pm 0.02$ & $0.38 \pm 0.03$ & 0.06680 & 0.00043 & 0.00004 \\
\hline $\begin{array}{l}\text { Food consump- } \\
\text { tion }(\mathrm{g})\end{array}$ & $>8$ & $124.52 \pm 7.54$ & $116.79 \pm 2.91$ & 0.7332 & $142.01 \pm 9.99$ & $127.85 \pm 3.01$ & 0.25107 & 0.22265 & 0.22284 \\
\hline $\begin{array}{l}\text { RW activity } \\
\text { (rounds/ } 3 \text { weeks) }\end{array}$ & $>19$ & $91,760.5 \pm 9292.9$ & $85,651.1 \pm 6134.6$ & 0.9578 & $101,535.9 \pm 10,343.6$ & $94,520.4 \pm 8245.3$ & 0.94397 & 0.89926 & 0.8239 \\
\hline
\end{tabular}

Voluntary running wheel activity and food consumption of DUhTP and DUC mice of individual generations were determined from day 49 to 70. Mice were kept in semi-barrier (SB) and SPF condition. All values are means \pm SE; $P$ values as indicated; significant differences are printed in bold typeface

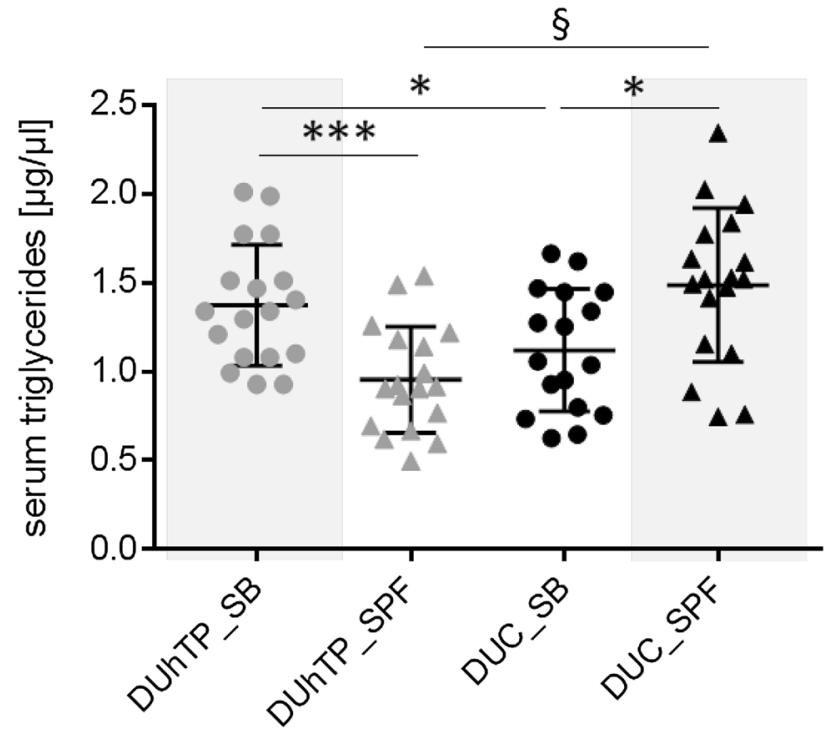

Fig. 4 Concentrations of triglycerides in serum of 70-day-old male DUhTP (grey data points) and DUC mice (black data points). Mice were kept in a semi-barrier (SB; dots) or in an SPF facility (triangles). Data are presented as means $\pm \mathrm{SE}(* P<0.05, * * * P<0.0005$, § $P<0.0001)$

\section{Effects of animal husbandry on the IGF system}

With respect to the differential effects of animal husbandry on body weight, we assessed serum concentrations of IGF-1 and IGFBPs in both mouse lines maintained under semi-barrier and SPF conditions. However, no significant differences of IGF-1 or IGFBP-2, -3 , and -4 could be observed (Table 3 ). In all conditions assessed, serum concentrations of IGF-1 and IGFBPs were highly variable in both mouse lines.

\section{Effects of animal husbandry on running performance, physical activity, and food consumption}

SPF husbandry significantly increased submaximal running performance activity in both mouse lines $(P<0.0001)$ if compared to the semi-barrier (Fig. 5). In $\mathrm{DUC}_{\mathrm{SPF}}$ or DUhTP $_{\mathrm{SPF}}$ mice, an almost 100 or $40 \%$ increase was observed, respectively. In absolute terms, the increase of submaximal running performance in DUhTP $_{\mathrm{SPF}}$ mice was almost twofold if compared to $\mathrm{DUC}_{\mathrm{SPF}}$ mice (DUhTP $\mathrm{SPF}$ $\left.+1620 \mathrm{~m} ; \mathrm{DUC}_{\mathrm{SPF}}+836 \mathrm{~m}\right)$. Thus, $\mathrm{DUhTP}_{\mathrm{SPF}}$ mice were able to maintain their prominent phenotype of higher running capacities if compared to unselected control mice also under conditions of SPF husbandry $(P<0.00001)$. Voluntary physical activity in running wheels and food consumption, examined at an age between 49 and 70 days during semibarrier and SPF husbandry in both mouse facilities, were similar in all experimental groups (Table 2).

\section{Effect of food on the body weights}

Feeding semi-barrier diet (Altromin) under SPF conditions for two generations in a subpopulation resulted in significantly reduced body weight in $\mathrm{DUC}_{\mathrm{SPF}}$ mice $(P<0.0001)$ and as a tendency also in DUhTP $\mathrm{SPF}_{\mathrm{SP}}$ mice (not significant) at an age of 21 days compared to the Ssniff diet (Fig. 6). While Ssniff-fed DUC mice had a mean body mass of $14.3 \mathrm{~g}$ corresponding to the weights of animals in SPF husbandry $(15.28 \pm 0.18 \mathrm{~g})$, body weights of Altromin-receiving mice were decreased to an average mass of $11.98 \mathrm{~g}$. Therefore, the body weights correspond to those of former animals in semibarrier husbandry $\left(\mathrm{DUhTP}_{\mathrm{SB}} 11.25 \pm 0.22 \mathrm{~g}\right.$; generation 149). Altromin diet in DUhTP mice increased TG, whereas 
Table 3 Serum concentrations of IGF-1, IGFBP-3, IGFBP-2, IGFBP-4 and the ratio between IGFBP-3/IGFBP-2 in 70-day-old DUhTP and DUC mice

\begin{tabular}{|c|c|c|c|c|c|c|c|c|c|}
\hline & $n$ & DUhTP_SB & DUhTP_SPF & $P$ & DUC_SB & DUC_SPF & $P$ & $\begin{array}{l}P \text { (DUhTP_ } \\
\text { SB versus } \\
\text { DUC_SB) }\end{array}$ & $\begin{array}{l}P \text { (DUhTP_ } \\
\text { SPF versus } \\
\text { DUC_SPF) }\end{array}$ \\
\hline $\begin{array}{l}\text { IGF1 (ng/ } \\
\mathrm{ml})\end{array}$ & $>16$ & $308.66 \pm 70.58$ & $168.38 \pm 49.91$ & 0.12603 & $192.92 \pm 57.10$ & $268.55 \pm 74.75$ & 0.44114 & 0.22510 & 0.36204 \\
\hline $\begin{array}{r}\text { IGFBP-3 } \\
(\mathrm{ng} / \mathrm{ml})\end{array}$ & $>16$ & $1171.43 \pm 248.59$ & $769.28 \pm 168.81$ & 0.20437 & $683.12 \pm 141.18$ & $875.99 \pm 167.67$ & 0.39937 & 0.10963 & 0.68958 \\
\hline $\begin{array}{r}\text { IGFBP-2 } \\
(\mathrm{ng} / \mathrm{ml})\end{array}$ & $>16$ & $435.76 \pm 79.02$ & $344.10 \pm 67.90$ & 0.40100 & $383.36 \pm 68.08$ & $367.25 \pm 61.6$ & 0.86575 & 0.62908 & 0.70329 \\
\hline $\begin{array}{r}\text { IGFBP-4 } \\
(\mathrm{ng} / \mathrm{ml})\end{array}$ & $>16$ & $193.35 \pm 30.42$ & $200.62 \pm 28.29$ & 0.86884 & $165.33 \pm 24.43$ & $258.49 \pm 51.32$ & 0.12853 & 0.49974 & 0.40422 \\
\hline $\begin{array}{l}\text { IGFBP-3/ } \\
\text { IGFBP-2 }\end{array}$ & $>16$ & $2.90 \pm 0.44$ & $2.44 \pm 0.48$ & 0.50454 & $1.89 \pm 0.33$ & $3.61 \pm 1.05$ & 0.12490 & 0.08496 & 0.37222 \\
\hline
\end{tabular}

Animals were kept in semi-barrier (SB) and SPF husbandry, respectively. All values are means \pm SE. $P$ values as indicated

Fig. 5 Long-term assessment of submaximal running capacity in male DUhTP (grey) and DUC mice (black) at an age of 70 days. Between generation 110 and 117 for line DUhTP or between generation 154 and 159 for line DUC, respectively, the animals were kept in semi-barrier conditions (dots). Running performance of DUhTP mice after generation 120 or of DUC mice after 165 generations was assessed in mice maintained under SPF conditions (triangles). Data are presented as means $\pm \mathrm{SE}(\S P<0.0001)$

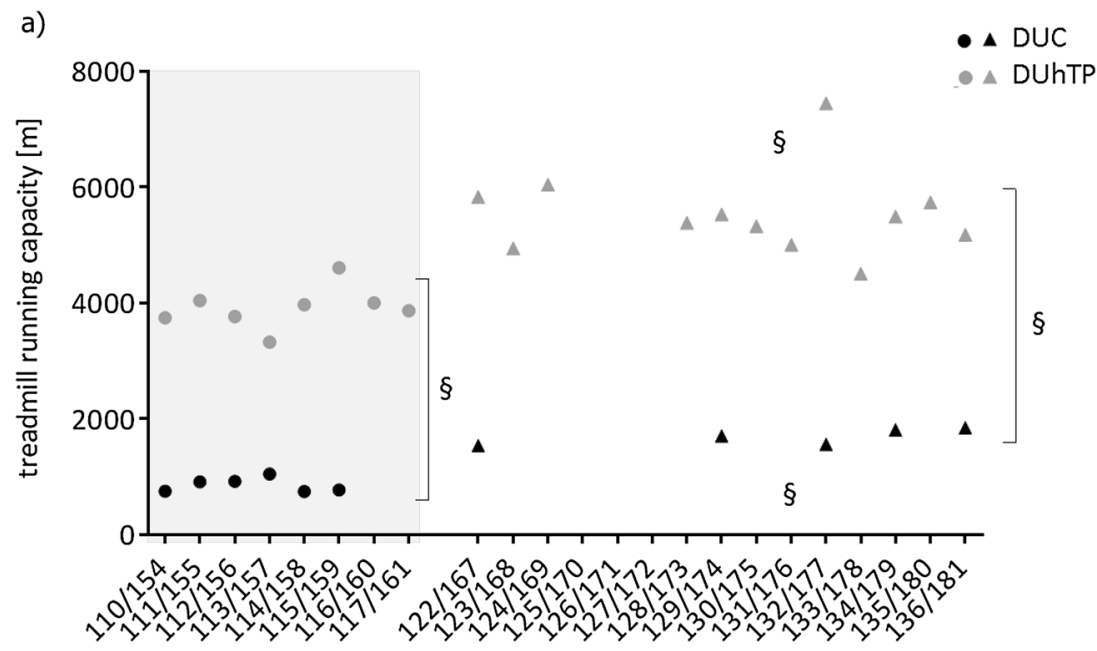

in DUC mice the same diet reduced the concentrations of TG in serum if compared to autoclaved Ssniff diet (Fig. 7).

\section{Discussion}

The Dummerstorf high treadmill performance mouse model DUhTP established by long-term selection for high treadmill performance is characterized by highly efficient fat mobilization (Brenmoehl et al. 2015), increased fat cell browning, and higher surface temperature (Brenmoehl et al. 2016) if compared to DUC mice deriving from the identical genetic background at the beginning of the selection experiment. Under conditions of Altromin1314 diet in the semi-barrier, DUhTP mice had a significant lower body mass than controls at day 70 but not at day 49 of age (Brenmoehl et al. 2013, 2015). After hygienic reorganization and establishing of Dummerstorf mouse lines in a new building, defined by the SPF status, we observed line-specific effects of the new environment. Due to the huge dimensions of the selection experiment-spanning more than 35 years now-and concerning the non-inbred structure of the genetic background-maintained by inclusion of up to 100 families per litter-the current phenotype of the mouse model requires an update and a discussion of potential effects due to the relocation or SPF husbandry in DUhTP versus DUC mice.

\section{Body and organ weights}

As a major effect of relocation or SPF husbandry, body weights and lengths were increased in $\mathrm{DUC}_{\mathrm{SPF}}$ mice to a significantly higher dimension if compared to DUhTP ${ }_{\mathrm{SPF}}$ mice. The stronger effects of SPF husbandry were evident in mice older than 5 days, when $\mathrm{DUC}_{\mathrm{SPF}}$ mice have higher body weights due to an increased body weight gain than DUhTP $_{\mathrm{SPF}}$ mice. It could be assumed that the higher living weight of the DUC mothers offers a better rearing capacity 


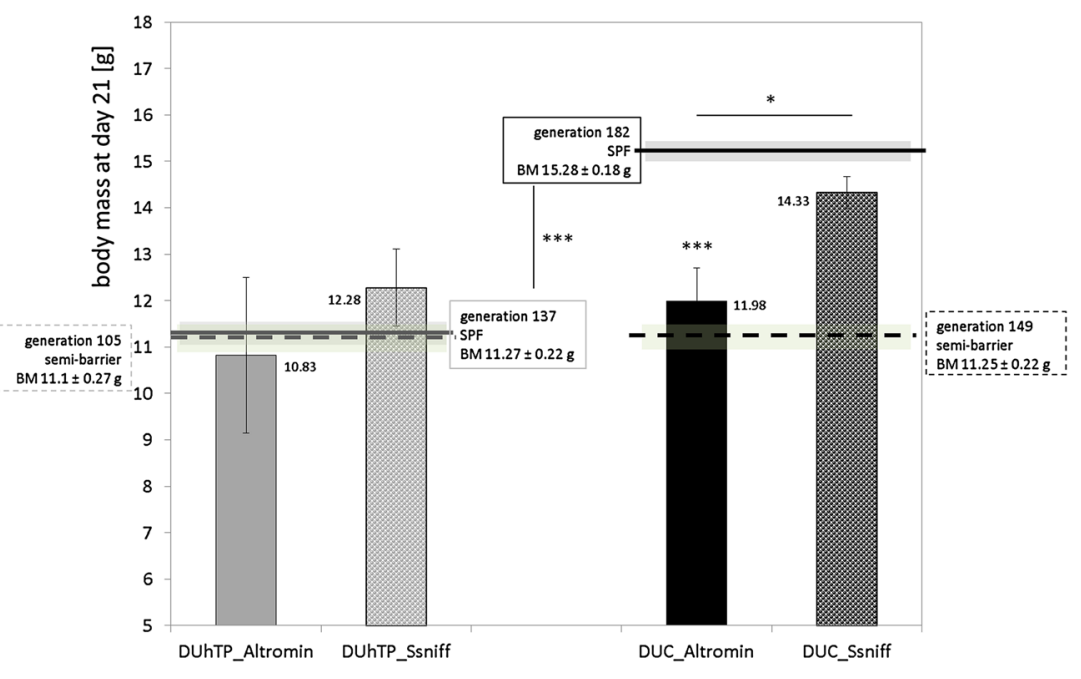

Fig. 6 Effect of diet on body mass in male DUhTP mice (grey bars) and unselected controls (DUC; black bars) maintained under SPF conditions at an age of 21 days. For the experiment, mice of both lines were kept in the quarantine station and either received Altromin diet fed in the semi-barrier or autoclaved Ssniff ${ }^{\circledR}$ diet fed in the SPF facility. The dashed line indicates body weights under conditions of semi-barrier husbandry (DUhTP: generation 105; DUC: generation 149). The continuous line displays body weights monitored within the SPF facility (DUhTP: generation 137; DUC: generation 182). Data are presented as means $\pm \operatorname{SE}(* P<0.05, * * * \mathrm{P}<0.0005)$
Fig. 7 Effect of diet on concentrations of triglycerides in serum obtained from male DUhTP (grey) and DUC mice (black data points) at an age of 70 days. Mice were kept in the quarantine station under SPF conditions and received SB (Altromin, open dots) and autoclaved SPF diet (Ssniff, open triangles), respectively. Data are presented as means $\pm \mathrm{SE}$ $\left({ }^{*} P<0.05, \S P<0.0001\right)$

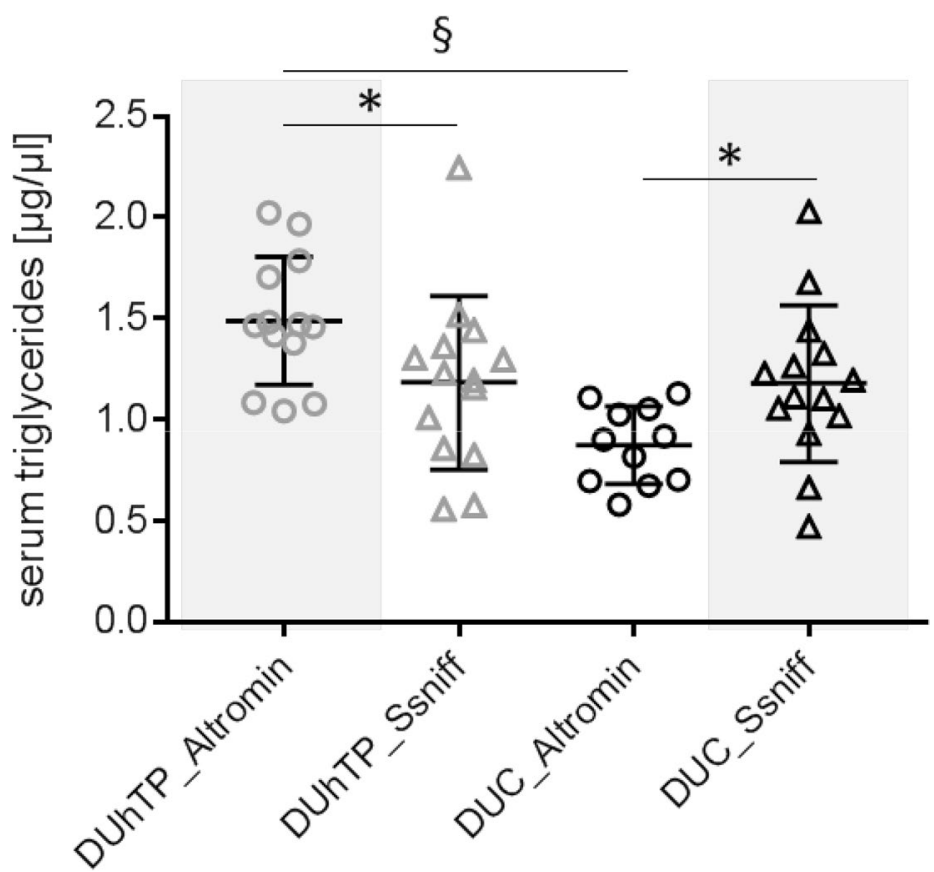

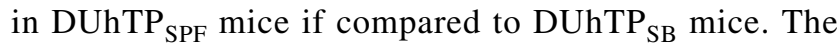
differential effects of animal husbandry on fat mass in both mouse lines were reflected also on the level of serum triglycerides, which were decreased by SPF in DUhTP but increased in DUC mice maintained under SPF conditions. For the increase of body weight in both mouse lines, we thus have to consider dietary factors such as food intake and macronutrient composition (Hall and Guo 2017). More generally, food intake or the input of metabolizable energy bandry. By contrast, epididymal fat mass was decreased 
were similar in both genetic lines and in both treatment groups at an age of 70 days $\left(\right.$ DUhTP $_{\mathrm{SB}} 831.3 \pm 89.0 \mathrm{kcal}$, DUhTP $_{\mathrm{SPF}} 787.9 \pm 42.9 \mathrm{kcal}$ and $\mathrm{DUC}_{\mathrm{SB}} 858.9 \pm 36.5 \mathrm{kcal}$, DUC $\left._{\text {SPF }} 812.7 \pm 99.8 \mathrm{kcal}\right)$. However, Ssniff diet contained more metabolizable energy from protein than Altromin (Tabel 2; Ssniff 36\%; Altromin 27\%). Accordingly, the expense of energy metabolizable from fat (Ssniff 11\%; Altromin 13\%) or of carbohydrates (Ssniff 53\%; Altromin $60 \%$ ) was higher in Altromin food. In adult mice, the use of high-protein diets can promote weight loss via reduction of fat accretion (Petzke et al. 2014). In the growth period, amino acids have been provided to highly efficient support growth rates in rats (Rogers and Harper 1965). In rats, direct comparison of a high versus low protein diet during pregnancy and lactation revealed higher growth rates in the offspring in a sex-specific manner (ThoneReineke et al. 2006). The protein-to-energy ratio also was demonstrated in non-rodent animals as an important determinant of weight gain (Garling and Wilson 1976; Dong et al. 2017). An excess of protein intake in mice by means of a $40 \%$ protein diet, impaired postnatal growth (Kucia et al. 2011), supporting a major effect of macronutrient composition also in mice. In addition to diet under SPF husbandry, also an improved animal health status due to the absence of defined pathogens has to be considered. To specifically address the effects of Ssniff versus Altromin diet, we applied both diets under SPF conditions over a period of two generations in a subpopulation. In fact, Altromin diet significantly reduced body weight in DUC mice in the absence of specific pathogens under conditions of SPF husbandry. Accordingly, body weights in DUC mice were no longer different if both treatments were compared identifying diet as the causative factor for the prominent weight increases and not the improved health status. In addition, feeding Altromin diet under SPF conditions reversed TG levels in both mouse lines resulting in a relative increase in DUhTP mice but in a decrease in DUC mice. Interestingly, increased protein intake by use of the earlier mentioned high-protein diet impaired liver weights (Kucia et al. 2011) and regulated hepatic expression of the GH/IGF pathway in the perinatal period in mice (Vanselow et al. 2011). In DUC $_{\mathrm{SPF}}$ mice, liver weights were unaffected by husbandry in both mouse lines. Because liver is a sensitive target for the effects of growth hormone (GH) (Bates et al. 1964), we may assume that accelerated growth in DUC mice in response to SPF husbandry is not related to altered $\mathrm{GH}$. This assumption is further supported by similar serum concentrations of IGF-1 and IGFBP-3 in all experimental conditions. Both IGF-I and IGFBP-3 represent established biomarkers for GH secretion (Blum et al. 1993). Also, the serum levels of IGFBP-2 were similar in both mouse lines and during semi-barrier or SPF husbandry. Because IGFBP-2 is potently suppressed by
GH, IGFBP-2 represents an indirect marker of GH secretion (Kirsch et al. 2007). Finally, the ratio of IGFBP-3/ IGFBP-2 as the most sensitive marker of GH secretion (Mesotten 2008) was unaffected by genotype or treatment, which further did not support effects of diet on GH secretion in our experimental system.

The accretion of muscle mass or body fat is related to physical activity or food consumption. However, both parameters also were similar both in DUC mice and in DUhTP mice independent of husbandry.

\section{Litter size and birth weight}

Relocation of DUC and DUhTP mice from the semi-barrier to the SPF facility increased the average litter size in DUC $_{\mathrm{SPF}}$ mice but not in DUhTP $\mathrm{SPF}_{\mathrm{SP}}$ mice. Litter size is a function of inbreeding (Falconer 1960), and inbreeding negatively affects litter size. Two arguments do not support an effect of inbreeding on the increase of litter size in $\mathrm{DUC}_{\mathrm{SPF}}$ mice. On one hand, during relocation not all DUC families could be transferred resulting in a reduction of genetic complexity in DUC mice. In addition, the increase in litter size in DUC mice was sudden and appears to be stable, which also may argue against a long-term effect of outbreeding. Due to the high number of founder animals in DUC with 110 and in DUhTP with 46 mice during relocation process in both mouse strains, we included a sufficient genetic background for phenotypic variation in different selection traits. The inbreeding level which could affect the fertility traits like litter size (Holt et al. 2004) has increased during the long selection period in both environments (DUC 0.175 and DUhTP 0.592, respectively). But no abrupt rise of the average inbreeding level could be observed after changing to the new mouse facility with SPF hygienic standard. Thus, we suppose the heavier body mass of $\mathrm{DUC}_{\mathrm{SPF}}$ mothers as a substantial factor or the increasing average number of born pups per litter. Those positive correlation between female body mass at mating and litter size were described by Holt et al. (2004). In addition, diets may have effects on reproductive performance including litter size (Ghosh et al. 2016) and an interaction between diet and genotype has been described in growth selected mice (Corva et al. 2004). Notably, diets with positive effects on body weight also increased reproductive performance in mice (Hoover-Plow et al. 1988), suggesting that the increases of body weight and litter size in $\mathrm{DUC}_{\mathrm{SPF}}$ mice are connected. Also, in other mammalian species, dietary factors have been identified with an effect on litter size (Gado et al. 2016; Li et al. 2015). Under field conditions, a link between food quality and reproductive performance could not be observed in mice (Ylonen et al. 2003).

Probably due to increased litter size, $\mathrm{DUC}_{\mathrm{SPF}}$ mice had lower body weights at birth than DUhTP $_{\mathrm{SPF}}$ mice. Between days 1 and days 21 after birth, $\mathrm{DUC}_{\mathrm{SPF}}$ mice were 
characterized by significantly increased weight gain if compared to DUhTP $_{\mathrm{SPF}}$ mice resulting in higher body weights in DUC mice elder than 5 days if compared to DUhTP mice. The increased growth rate of $\mathrm{DUC}_{\mathrm{SPF}}$ mice in the perinatal period may thus be due to accelerated compensatory growth or due to SPF husbandry. Very clearly, also in mice neonatal catch-up growth shortly after birth is associated with visceral obesity later in life (Isganaitis et al. 2009). Thus, reduced birth weight in fact may trigger catch-up growth and visceral obesity, and therefore contribute at least in part to the markedly increased body weights in $\mathrm{DUC}_{\mathrm{SPF}}$ mice. However, in $\mathrm{DUC}_{\mathrm{SPF}}$ mice also muscle weight was increased if compared to $\mathrm{DUC}_{\mathrm{SB}}$ mice which is not explained by reduced birth weights or catch-up growth in the perinatal period because low birth weight was associated with increased fat mass but reduced lean mass in mice (Ju et al. 2016). Therefore, also an effect of maternal diet has to be considered which is well documented in the literature, e.g., by the postnatal control of growth or litter performance in suckling pigs (He et al. 2016). Finally, litter size is also a function of fetal mortality (Falconer 1960) and reproductive performance. In rats, the absence of pathogens under SPF conditions significantly increased litter size if compared to a conventional barrier, which was discussed in a context of higher numbers of corpora lutea and decreased post implantation losses of embryos or fetuses (Wakafuji et al. 1984).

\section{Effects of cryopreservation}

For the efficient resettlement and establishment of the new and empty mouse facility, embryos from $\mathrm{DUC}_{\mathrm{SB}}$ mice were cryoconserved by vitrification and transferred into pseudopregnant Crl:CD1(ICR) fosters defined by the SPF status. These generated $\mathrm{DUC}_{\mathrm{SPF}}$ mice were used for initial stocking of the new SPF facility in Dummerstorf. Additionally, fresh embryos from line $\mathrm{DUC}_{\mathrm{SB}}$ were transferred to pseudopregnant $\mathrm{CD} 1$ and $\mathrm{DUC}_{\mathrm{SFP}}$ foster mothers. By contrast, fresh embryos from DUhTP $_{\mathrm{SB}}$ mice were used for transfer without cryopreservation. Therefore, we cannot exclude that cryoconservation in $\mathrm{DUC}_{\mathrm{SPF}}$ mice is related to differential phenotype effects of SPF husbandry. In fact, higher body weights have been observed in cryopreserved mice if compared to controls (Dulioust et al. 1995). However, the increases in body weight were described as a long-term effect in adult mice only (Dulioust et al. 1995). Because growth activities were evident early after birth in $\mathrm{DUC}_{\mathrm{SPF}}$ mice, cryopreservation seems not to account for the differential growth response of DUC versus DUhTP mice during SPF husbandry. Cryopreservation in human embryos explicitly did not affect growth during infancy and early childhood (Wennerholm et al. 1998) also not arguing for an effect of cryoconservation in young $\mathrm{DUC}_{\mathrm{SPF}}$ mice. In general, it can be doubted that potential epigenetic modification established during handling of the embryo is transmitted to the offspring at all, because in cloned mice altered growth and obesity were found only in the born animal but not in the offspring of cloned mice (Tamashiro et al. 2002). In DUC $\mathrm{SPF}_{\mathrm{SP}}$ mice, the phenotype of elevated growth is observed over 15 generations now, not supporting reversible effects of epigenetic modifications.

\section{Effects on running capacity}

Animal husbandry in SPF conditions increased absolute weights of body, muscle, and different fat depots in DUC but not in DUhTP mice. These specific effects were not reflected by differential effects on running capacities in DUhTP mice versus DUC mice. Instead, SPF husbandry significantly elevated running capacities in both lines $(P<0.0001)$. Therefore, we may assume that the positive effects of husbandry on endurance exercise are related to improved animal health, due to the lack of specific pathogens and/or to the diet fed in the new SPF facility. Some pathogens (e.g., Helicobacter spp. or Pasteurellaceae) included in hygiene monitoring according to the FELASA guidelines (Mahler Convenor et al. 2014) are less clinically apparent. Under conditions of stress or in the presence of coinfections, clinical signs may become more prominent. Infections with mouse hepatitis virus (MHV) or the presence of endo- and ectoparasites severely can impact on animal health and result in lifethreatening tissue damage during acute infection (Baker 2003). Because latent infections form various pathogens could be present under conditions of semi-barrier husbandry, it is conceivable that exercise capacity was limited in $\mathrm{DUC}_{\mathrm{SB}}$ and DUhTP ${ }_{\mathrm{SB}}$ mice by coinfection with different pathogens.

\section{Summary and conclusion}

Here, we present a very rare direct comparison of different husbandry conditions on physiological parameters in animal models. The small number of comparative analyses, that can be found in the literature, relates to questions on health status in pigs (Clapperton et al. 2009), effects on immune state in mice (Beura et al. 2016), decontamination of mice (Wiese et al. 2007), or enzyme activity of alkaline phosphatase (Pickering and Pickering 1984). In the present comparative analysis, we demonstrate that both improved health and diet used under SPF conditions have multiple effects in the DUC/DUhTP mouse model. DUC $\mathrm{SPF}_{\mathrm{SP}}$ mice have higher body weights at an age of more than 5 days and this effect is almost exclusively an effect of diet. The increased litter size might be an effect both of body weight and improved health and could be responsible for reduced birth weights in $\mathrm{DUC}_{\mathrm{SPF}}$ mice. The strong increases of running capacities observed in both mouse lines could represent a benefit of 
improved health status in both mouse lines due to the lack of pathogens. However, very clearly we provide evidence that DUC and DUhTP mice differentially respond to environmental factors including improved health and an adjusted diet. From the differential increase or decrease of body fat, we may assume that energy metabolism is involved in the differential response of both mouse lines to environmental factors. Our results therefore strongly support the interaction of genomes with the environment, which may inspire the inclusion of environmental factors in phenotype analysis performed in animal models.

Acknowledgements The authors want to thank Luong Chau, Dr. Daniela Ohde, Zianka Meyer and Sabine Hinrichs, Sabine Maibohm, Benita Lucht, Karin Ullerich, Sonja Alm, Dr. Ulla Renne, Erika Wytrwat, and Ines Müntzel for excellent technical assistance. The publication of this article was funded by the Open Access Fund of the Leibniz Institute for Farm Animal Biology (FBN).

\section{Compliance with ethical standards}

Conflict of interest All authors declare no conflicts of interest.

Open Access This article is distributed under the terms of the Creative Commons Attribution 4.0 International License (http://creativecommons.org/licenses/by/4.0/), which permits unrestricted use, distribution, and reproduction in any medium, provided you give appropriate credit to the original author(s) and the source, provide a link to the Creative Commons license, and indicate if changes were made.

\section{References}

Baker DG (2003) Natural pathogens of laboratory animals: their effects on research. ASM Press, Washington, DC

Bates RW, Milkovic S, Garrison MM (1964) Effects of prolactin, growth hormone and acth, alone and in combination, upon organ weights and adrenal function in normal rats. Endocrinology 74:714-723

Beura LK et al (2016) Normalizing the environment recapitulates adult human immune traits in laboratory mice. Nature 532(7600):512-516

Blum WF et al (1993) Serum levels of insulin-like growth factor I (IGF-I) and IGF binding protein 3 reflect spontaneous growth hormone secretion. J Clin Endocrinol Metab 76(6):1610-1616

Brenmoehl J et al (2013) Metabolic adaptations in the liver of born long-distance running mice. Med Sci Sports Exerc 45(5):841-850

Brenmoehl J et al (2015) Dynamics of fat mass in duhtp mice selected for running performance-fat mobilization in a walk. Obes Facts $8(6): 373-385$

Brenmoehl J et al (2016) Browning of subcutaneous fat and higher surface temperature in response to phenotype selection for advanced endurance exercise performance in male DUhTP mice. J Comp Physiol B 187:361-373

Clapperton $\mathrm{M}$ et al (2009) Traits associated with innate and adaptive immunity in pigs: heritability and associations with performance under different health status conditions. Genet Sel Evol 41:54

Corva PM et al (2004) Diet effects on female reproduction in high growth (hg/hg) mice that are deficient in the Socs-2 gene. Reprod Nutr Dev 44(4):303-312
Dietl G, Langhammer M, Renne U (2004) Model simulations for genetic random drift in the outbred strain Fzt:DU. Arch Tierzucht 47:595-604

Dong LF et al (2017) Feeding different dietary protein to energy ratios to Holstein heifers: effects on growth performance, blood metabolites and rumen fermentation parameters. J Anim Physiol Anim Nutr (Berl) 101(1):30-37

Dulioust E et al (1995) Long-term effects of embryo freezing in mice. Proc Natl Acad Sci USA 92(2):589-593

Falconer DS (1960) The genetics of litter size in mice. J Cell Comp Physiol 56(Suppl 1):153-167

Falkenberg H, Langhammer M, Renne U (2000) Comparison of biochemical blood traits after long-term selection on high or low locomotory activity in mice. Arch Tierzucht 43:513-522

Gado HM et al (2016) Fertility, mortality, milk output, and body thermoregulation of growing Hy-Plus rabbits fed on diets supplemented with multi-enzymes preparation. Trop Anim Health Prod 48(7):1375-1380

Garling DL Jr, Wilson RP (1976) Optimum dietary protein to energy ratio for channel catfish fingerlings, Ictalurus punctatus. J Nutr 106(9):1368-1375

Ghosh $\mathrm{S}$ et al (2016) Severe but Not moderate vitamin B12 deficiency impairs lipid profile, induces adiposity, and leads to adverse gestational outcome in female C57BL/6 mice. Front Nutr 3:1

Hall KD, Guo J (2017) Obesity energetics: body weight regulation and the effects of diet composition. Gastroenterology 152:1718-1727

$\mathrm{He} \mathrm{B}$ et al (2016) Effects of sodium butyrate supplementation on reproductive performance and colostrum composition in gilts. Animal 10(10):1722-1727

Holt M, Vangen O, Farstad W (2004) Components of litter size in mice after 110 generations of selection. Reproduction 127(5):587 -592

Hoover-Plow J, Elliott P, Moynier B (1988) Reproductive performance in C57BL and I strain mice. Lab Anim Sci 38(5):595-602

Isganaitis E et al (2009) Accelerated postnatal growth increases lipogenic gene expression and adipocyte size in low-birth weight mice. Diabetes 58(5):1192-1200

$\mathrm{Ju} \mathrm{L}$ et al (2016) Endurance exercise ameliorates low birthweight developed catch-up growth related metabolic dysfunctions in a mouse model. Endocr J 63(3):275-285

Kirsch S et al (2007) Development of an absolute quantification method targeting growth hormone biomarkers using liquid chromatography coupled to isotope dilution mass spectrometry. J Chromatogr A 1153(1-2):300-306

Kucia M et al (2011) High-protein diet during gestation and lactation affects mammary gland mRNA abundance, milk composition and pre-weaning litter growth in mice. Animal 5(2):268-277

Li J et al (2015) Effects of arginine supplementation during early gestation (day 1 to 30 ) on litter size and plasma metabolites in gilts and sows. J Anim Sci 93(11):5291-5303

Mahler Convenor M et al (2014) FELASA recommendations for the health monitoring of mouse, rat, hamster, guinea pig and rabbit colonies in breeding and experimental units. Lab Anim 48(3): 178-192

Mesotten G (2008) Changes within the GH/IGF-I/IGFBP axis in critical illness. In: Berghe GVD (ed) Contemporary endocrinology: acute cause to consequence. Humana Press, New York, pp 181-199

Petzke KJ, Freudenberg A, Klaus S (2014) Beyond the role of dietary protein and amino acids in the prevention of diet-induced obesity. Int J Mol Sci 15(1):1374-1391

Pickering CE, Pickering RG (1984) Alkaline phosphatase activity of the mouse. Comp Biochem Physiol C 79(2):417-424

Rogers QR, Harper AE (1965) Amino acid diets and maximal growth in the rat. J Nutr 87(3):267-273

Tamashiro KL et al (2002) Cloned mice have an obese phenotype not transmitted to their offspring. Nat Med 8(3):262-267 
Thone-Reineke C et al (2006) High-protein nutrition during pregnancy and lactation programs blood pressure, food efficiency, and body weight of the offspring in a sex-dependent manner. Am J Physiol Regul Integr Comp Physiol 291(4):R1025-R1030

Vanselow J et al (2011) Hepatic expression of the GH/JAK/STAT/IGF pathway, acute-phase response signalling and complement system are affected in mouse offspring by prenatal and early postnatal exposure to maternal high-protein diet. Eur J Nutr 50(8):611-623

Wakafuji Y et al (1984) Increase in litter size and decrease of postimplantation loss of fetuses observed in an SPF colony of WistarImamichi rats. Jikken Dobutsu 33(2):165-171

Wennerholm UB et al (1998) Postnatal growth and health in children born after cryopreservation as embryos. Lancet 351(9109):1085-1090
Wiese E et al (2007) Decontamination of a barrier facility using microisolator cages and provisional partitioning. Lab Anim (NY) 36(7):31-35

Wirthgen E et al (2016) Quantitative Western ligand blotting reveals common patterns and differential features of IGFBP-fingerprints in domestic ruminant breeds and species. Growth Horm IGF Res 26:42-49

Ylonen $\mathrm{H}$ et al (2003) Is reproduction of the Australian house mouse (Mus domesticus) constrained by food? A large-scale field experiment. Oecologia 135(3):372-377 